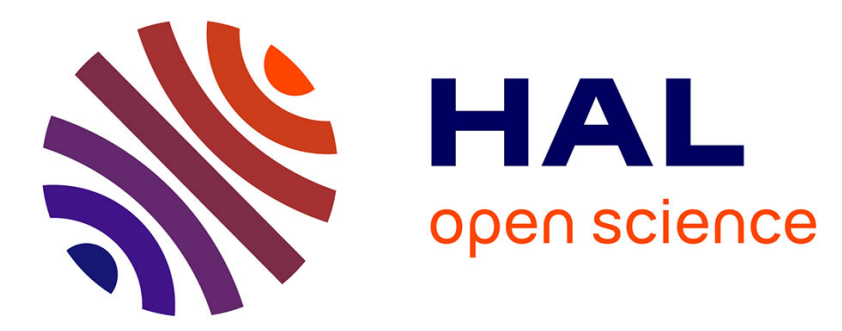

\title{
Observer-based Controller For Microrobot in Pulsatile Blood Flow
}

Lounis Sadelli, Matthieu Fruchard, Antoine Ferreira

\section{To cite this version:}

Lounis Sadelli, Matthieu Fruchard, Antoine Ferreira. Observer-based Controller For Microrobot in Pulsatile Blood Flow. IEEE Conference on Decision and Control, Dec 2014, Los Angeles, United States. hal-01071287

\section{HAL Id: hal-01071287 \\ https://hal.science/hal-01071287}

Submitted on 3 Oct 2014

HAL is a multi-disciplinary open access archive for the deposit and dissemination of scientific research documents, whether they are published or not. The documents may come from teaching and research institutions in France or abroad, or from public or private research centers.
L'archive ouverte pluridisciplinaire HAL, est destinée au dépôt et à la diffusion de documents scientifiques de niveau recherche, publiés ou non, émanant des établissements d'enseignement et de recherche français ou étrangers, des laboratoires publics ou privés. 


\title{
Observer-based Controller For Microrobot in Pulsatile Blood Flow
}

\author{
Lounis Sadelli, Matthieu Fruchard, Antoine Ferreira
}

\begin{abstract}
-
We propose an observer-based controller for a magnetic microrobot immersed in the human vasculature. The drag force depends on the pulsatile blood velocity and specially acts on the microrobot dynamics. In the design of advanced control laws, the blood velocity is usually assumed to be known or set to a constant mean value to achieve the control objectives, whereas the sole robot position is measured. We prove the stability of the proposed observer-based controller combining a backstepping controller with a mean value theorem (MVT) based observer. The resulting estimation of the blood velocity is then illustrated and compared to high gain observer results through simulations.
\end{abstract}

\section{INTRODUCTION}

Therapeutic untethered nano and microrobots control has been an ongoing interest for many researchers since such systems can perform complex surgical procedures and reach remote places with lessened medical side effects [1], [2]. Different propulsion strategies have been proposed, mainly based on magnetic deported actuation: robots with elastic flagellum [3], [4], [5], with helical flagellum [6], [7], bead pulled robots or swarm of robots [8], [9].

Whatever the proposed design, these systems face nonlinear forces: blood drag, electrostatic force, etc [10], [11]. Among these forces, the nonlinear drag force both prevails at a small scale and is the most disturbed by external time-varying perturbations because of the pulsatile blood flow. Its measurement is often assigned to ultrasonic sensors [12] or magnetic resonance imaging devices [13] that exploit the Doppler effect. Yet this solution calls for an end-effector servoing so as to track the robot. Moreover, the devices resolution is not compatible with the precision required to discriminate the spatial parabolic blood flow profile (see Fig 1) the robot faces depending on its position in the vessel. Another solution relies on a priori knowledge of the blood velocity, either using computational solutions of the Navier Stokes equation [14], or analytical expressions of the blood velocity profiles [10]. However, the former is not well suited for real-time purposes, whilst the latter requires a precise a priori knowledge of the vessel geometry. Considering the blood flow as a disturbance to reject it is neither a good idea since drag is the dominant force: blood velocity is thus relevant for control purposes. To

L. Sadelli and M. Fruchard are with the Laboratory PRISME EA 4229, Univ. Orléans, 63 Av de Lattre de Tassigny, 18020 Bourges Cedex, France, lounis.sadelli@univ-orleans.fr matthieu.fruchard@univ-orleans.fr

A. Ferreira is with the Laboratory PRISME EA 4229, INSA-CVL, 88 Bd Lahitolle, 18000, Bourges, France. antoine.ferreiradinsa-cvl.fr

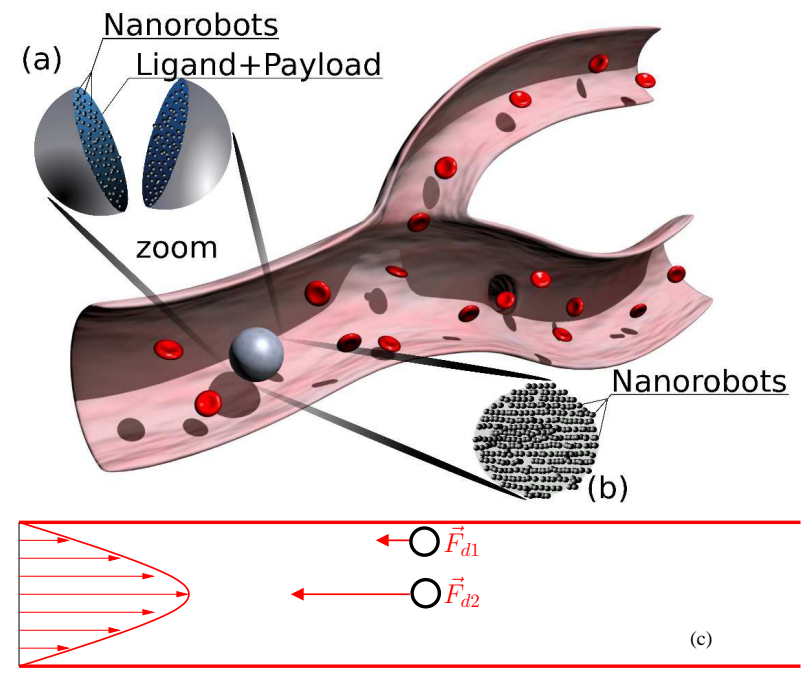

Fig. 1. Microrobot in a blood vessel. (a) binded by ligand, including a payload; (b) binded by magnetic and interaction forces. (c) The microrobots do not face the same drag force because of the parabolic blood flow profile.

avoid the drawbacks of the aforementioned approaches, we have consequently proposed in [15] a high gain observer to estimate the blood velocity and inject it in the control law. Yet, high gain observers are known for their output noise sensitivity, especially as the system dimension increases, as underlined in [16].

The purpose of this paper is to propose an alternative state estimation of the blood velocity using a MVT-based observer so as to both improve robustness to noise and prove the stability of the observer-based controller. The model of the microrobot dynamics taking into account the blood velocity modeled by a truncated Fourier series is first recalled in Section II. Section III is dedicated to the design of a stable MVT observer-based backstepping controller. Finally, simulations results illustrate the efficiency of the proposed approach and are compared to the high gain observer results in Section IV. Conclusions and discussions on open issues are summarized in Section V.

\section{MODELING}

Let us consider a microrobot of mass $m$, made of ferromagnetic particles and a payload, navigating in the arterial network. Any system immersed in a moving fluid is -at leastsubjected to two forces: the drag force and the buoyancy force. This section is devoted to briefly introduce these forces and the magnetic motive force (see e.g. [10] for more details). For sake of simplicity, we here only consider a $1 D$ model. 


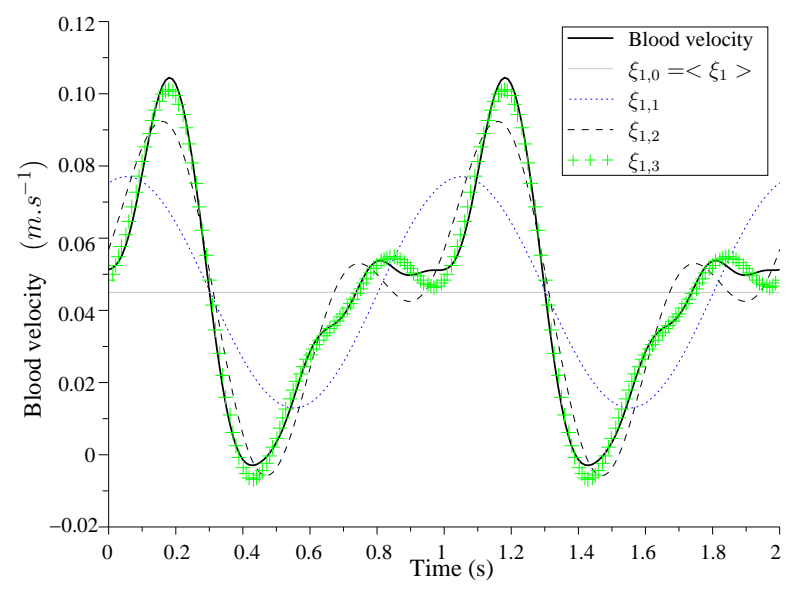

Fig. 2. Blood velocity $\xi_{1}$ (black solid line), mean value $\xi_{1,0}$ (gray solid line) and $n$th order truncated Fourier series $\xi_{1, n}: \xi_{1,1}$ (blue dots), $\xi_{1,2}$ (black dashes) and $\xi_{1,3}$ (green crosses).

\section{A. Forces}

1) Hydrodynamic drag force: In a fluidic environment, any system faces the drag force which opposes its motion:

$$
\left\{\begin{aligned}
\vec{F}_{d} & =-\frac{1}{2} \rho_{f} A C_{d}\left(\frac{\|\vec{v}\|}{\beta}\right)^{2} \frac{\vec{v}}{\|\vec{v}\|} \\
C_{d} & =\frac{24}{R e}+\frac{6}{1+\sqrt{R e}}+0.4 \\
R e & =\frac{2 r \rho_{f}\|\vec{v}\|}{\beta \eta}
\end{aligned}\right.
$$

where $A$ is the frontal area, the drag coefficient $C_{d}$ is given by [17], $\beta$ is a dimensionless ratio related to the wall effect caused by the partial vessel occlusion by the robot [18], and $v$ denotes the relative velocity between the robot and the fluid. $\eta$ and $\rho_{f}$ denote respectively the blood viscosity and density. In the case of a spherical microrobot of radius $r$, the drag force can be rewritten as:

$$
\vec{F}_{d}=-\left(a|v|+b v^{2}+c \frac{v^{2}}{1+d \sqrt{|v|}}\right) \frac{\vec{v}}{\|\vec{v}\|}
$$

The parameters in (2) are inherited from (1):

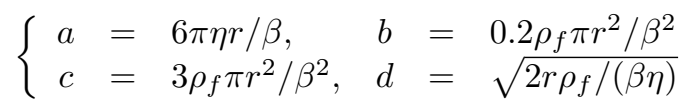

Arterial pulsatile flow profiles are usually modeled using the Womersley model [19], which results in a truncated Fourier series as shown on Fig. 2. It is easy to show that any blood velocity $\xi_{1}$ expressed as a $n$th order truncated Fourier series is solution of the autonomous system:

$$
\left(\mathcal{S}_{\xi}\right) \quad\left\{\begin{array}{cl}
\dot{\xi}_{2 k-1} & =\xi_{2 k} \\
\dot{\xi}_{2 k} & =-\omega^{2}\left(k^{2} \xi_{2 k-1}-\xi_{2 k+1}\right) \\
\vdots & \\
\dot{\xi}_{2 n+1} & =0
\end{array}\right.
$$

$\forall k \in\{1, \ldots, n\}$ and where the mean value is $\xi_{2 n+1}$, up to a constant factor $\prod_{i=1}^{n} i^{2}$. See [15] for technical details.
2) Magnetic force: Three main propulsion designs for magnetic microrobots have been developped: bead pulled, elastic flagellated, and helical tailed robots, see [1] for a survey. In $1 \mathrm{D}$, their magnetic motive force is given by [10]:

$$
\vec{F}_{m}=\beta_{2} \vec{u}-\beta_{3} \vec{v}
$$

where $\beta_{3}$ is related to the drag exerting on the helical tail, and is null for elastic flagellated and bead pulled robots. The control input $u$ is the magnetic field gradient $\nabla B$ and the frequency of the oscillating magnetic field $B$ for bead pulling and flagellated robots, respectively. $\beta_{2}$ is proportional to the robot magnetization, radius, and ferromagnetic ratio, denoted $\vec{M}, r$, and $\tau_{m}$ respectively.

3) Apparent weight: The apparent weight of the robot results from the contribution of the weight and the buoyancy:

$$
\vec{W}_{a}=V\left(\rho-\rho_{f}\right) \vec{g}
$$

where the robot density is $\rho=\tau_{m} \rho_{m}+\left(1-\tau_{m}\right) \rho_{p}$ with $\rho_{m}$ and $\rho_{p}$ the magnetic and payload densities, respectively. $V$ denotes the robot volume.

\section{B. State Space Representation}

The reduced system $\left(\mathcal{S}_{r}\right)$ is derived from (2), (5) and (6):

$$
\left(\mathcal{S}_{r}\right):\left\{\begin{array}{l}
\dot{z}=\left[\begin{array}{ll}
0 & 1 \\
0 & 0
\end{array}\right] z+\left[\begin{array}{c}
0 \\
\beta_{2}
\end{array}\right] u+\left[\begin{array}{c}
0 \\
\phi\left(\left(z^{T}, \xi^{T}\right)^{T}\right)
\end{array}\right] \\
y=C_{1} z
\end{array}\right.
$$

where the state $z$ components $z_{1}$ and $z_{2}$ denote respectively the robot position and velocity, $u$ the control input and $\xi_{1}$ the blood velocity. The output $y$ is the robot position measured by an imager, i.e. $C_{1}=\left[\begin{array}{ll}1 & 0\end{array}\right]$. The function $\phi$ is given by:

$$
\begin{aligned}
\phi(z, \xi)= & {\left[-\sigma\left(z_{2}-\xi_{1}\right)\left(\left(a+\beta_{3}\right)\left|z_{2}-\xi_{1}\right|+b\left(z_{2}-\xi_{1}\right)^{2}\right.\right.} \\
& \left.\left.+c \frac{\left(z_{2}-\xi_{1}\right)^{2}}{1+d \sqrt{\left|z_{2}-\xi_{1}\right|}}\right)+V\left(\rho_{f}-\rho\right) g\right] \frac{1}{m}
\end{aligned}
$$

where $\sigma$ is the sign function.

Let $x=\left[z^{T}, \xi^{T}\right]^{T} \in \mathbb{R}^{2 n+3}$ denote an extended state vector. The extended system $\left(\mathcal{S}_{e}\right)$ is inherited from (7) and (4):

$$
\left(\mathcal{S}_{e}\right):\left\{\begin{array}{l}
\dot{x}=A(0) x+B f(x, u) \\
y=C_{2} x
\end{array}\right.
$$

with $B^{T}=\left[\begin{array}{lll}0 & 1 & 0_{1 \times 2 n+1}\end{array}\right]$ and $C_{2}=\left[\begin{array}{ll}C_{1} & 0_{1 \times 2 n+1}\end{array}\right]$. Function $f$ is chosen so that $A(0)$ contains the linear part of $\left(\mathcal{S}_{e}\right)$ :

$$
\begin{array}{r}
f(x, u)=\phi(x)+\beta_{2} u+\frac{\left(a+\beta_{3}\right)\left(x_{2}-x_{3}\right)}{m} \\
A(\alpha)=\left[\begin{array}{ccccc}
\chi_{1} & \chi_{2} & O_{2 \times 1} & \cdots & O_{2 \times 1} \\
O_{2} & \digamma_{1} & \chi_{3} & \cdots & O_{2 \times 1} \\
\vdots & \ddots & \ddots & \ddots & \vdots \\
O_{2} & \cdots & O_{2 \times 1} & \digamma_{n} & \chi_{3} \\
0 & \cdots & \cdots & \cdots & 0
\end{array}\right]
\end{array}
$$


with

$$
\begin{array}{ll}
\chi_{1}=\left[\begin{array}{cc}
0 & 1 \\
0 & -\left(\beta_{3}+a\right) / m-\alpha
\end{array}\right] & \chi_{3}=\left[\begin{array}{c}
0 \\
\omega^{2}
\end{array}\right] \\
\chi_{2}=\left[\begin{array}{cc}
0 & 0 \\
\left(\beta_{3}+a\right) / m+\alpha & 0
\end{array}\right] & \digamma_{i}=\left[\begin{array}{cc}
0 & 1 \\
-i^{2} \omega^{2} & 0
\end{array}\right]
\end{array}
$$

The knowledge of $x_{1}, x_{2}, x_{3}$ is required to implement a stabilizing control law. Since only $x_{1}$ is measured, an observer is thus necessary. The next section is devoted to the synthesis of the observer-based controller.

\section{MAIN RESUlT}

Lemma 1 Let $z_{\text {ref }}=\left[z_{r}(t), \dot{z}_{r}(t), \ddot{z}_{r}(t)\right]$ denote any continuous and bounded reference trajectory, and $\mathcal{K}_{z}$ denote any compact subset of a neighborhood of $\left(z_{r}, \dot{z}_{r}\right) . \forall x \in \mathcal{K}=$ $\mathcal{K}_{z} \times \mathcal{K}_{\xi}$, systems (7) and (9) satisfies the properties:

P1) $\phi$ is locally $\gamma$-Lipschitz on $\mathcal{K}$;

P2) System (7) is locally controllable along $z_{\text {ref; }}$;

P3) System (9) satisfies $\left(A_{1}, C_{2}\right)$ and $\left(A_{2}, C_{2}\right)$ observable, with $A_{1}=A(0)$ and $A_{2}=A\left(\gamma-\frac{a+\beta_{3}}{m}\right)$;

P4) $f$ is differentiable with respect to $x$ and $\forall j \leq 2 n+3$, $\exists\left(a_{j}, b_{j}\right) \in \mathbb{R}^{2}$ such that:

$$
a_{j} \leq \frac{\partial f}{\partial x_{j}}(x, u) \leq b_{j} \quad \forall x \in \mathcal{K}, \forall u
$$

Proof: Properties (P1) and (P2) have been demonstrated in [15] and [20], respectively. In particular, the local controllability of system (7) is inherited from the controllability of its linearized time-variant system along the reference trajectory $z_{\text {ref }}(t)$ [21], [22].

Let $\Upsilon$ denote the observability matrix determinant associated with the pair $\left(C_{2}, A(\alpha)\right)$. It is straightforward that:

$$
\Upsilon=\left[\left(\left(\beta_{3}+a\right) / m+\alpha\right)^{2 n+1} \prod_{i=0}^{n-1} \omega^{4 i}\right] \omega^{2 n}
$$

Property (P4) is inherited from (10), from (P1) and from the properties of $\phi$ established in Lemma 1 of [15], namely $\frac{\partial \phi}{\partial x_{2}}=-\frac{\partial \phi}{\partial x_{3}}$ and $\forall x \in \mathcal{K}:$

$$
\frac{a+\beta_{3}}{m} \leq \frac{\partial \phi}{\partial x_{3}}(x) \leq \gamma
$$

Using (10) and (15), it is straightforward that:

$$
\begin{gathered}
0 \leq \frac{\partial f(x, u)}{\partial x_{3}}=-\frac{\partial f(x, u)}{\partial x_{2}} \leq \gamma-\frac{a+\beta_{3}}{m} \\
\frac{\partial f(x, u)}{\partial x_{j}}=0 \forall j \notin\{2,3\}
\end{gathered}
$$

\section{A. Observer}

We previously have proposed a high gain observer in [15] to estimate the extended state vector in (9). Yet this observer raises two issues. First, even for small dimensional systems, it is difficult to design the observer in order to obtain relatively low gain $L$ because of the high value of the local Lipschitz constant $\gamma$. Second, even for a blood velocity defined as a first-order truncated Fourier series, the system (9) dimension is 5. Using higher order truncated series emphasizes this issue since the gain matrix is formed in ascending powers of $L$. That is the reason why we propose another observer based on [23].

Proposition 1 Let $\mathcal{U}$ denote the compact set of admissible inputs. Under assumptions of Lemma (1), $\forall x(0) \in \mathcal{K}$, $\forall \hat{x}(0) \in \mathcal{K}, \forall u \in \mathcal{U}$,

$$
\left\{\begin{array}{l}
\dot{\hat{x}}=A(0) \hat{x}+B f(\hat{x}, u)+K_{o}\left(y-C_{2} \hat{x}\right) \\
\hat{y}=C_{2} \hat{x}
\end{array}\right.
$$

is an exponential observer of system (9) on $\mathcal{K}$ with the symmetric positive definite matrices $P_{o}, Q_{o}$ and the gain $K_{o}$ that satisfy the Linear Matrix Inequalities (LMI):

$\left(A_{i}-K_{o} C_{2}\right)^{T} P_{o}+P_{o}\left(A_{i}-K_{o} C_{2}\right)<-Q_{o}, \quad \forall i \in\{1,2\}$

Proof: Let $\tilde{x}=x-\hat{x}$ denote the estimation error. Let $C o(x, \hat{x})=\{\lambda x+(1-\lambda) \hat{x}, 0 \leq \lambda \leq 1\}$ and $\bar{C} o(x, \hat{x})$ denote a convex and its convex hull. Applying the MVT to $f, \exists \theta(t) \in \bar{C} o(x, \hat{x})$ such that:

$$
\tilde{f}=f(x)-f(\hat{x})=\left(\sum_{j=1}^{2 n+3} e_{j}^{T} \frac{\partial f}{\partial x_{j}}(\theta(t), u(t))\right) \tilde{x}(t)
$$

with $\left\{e_{1}, \ldots e_{2 n+3}\right\}$ denoting the canonical basis of $\mathbb{R}^{2 n+3}$.

Let $\mathcal{A}(h(t))=A(0)+B \sum_{j=1}^{2 n+3} h_{j}(t) e_{j}^{T} \quad$ with $h_{j}(t)=\frac{\partial f}{\partial x_{j}}(\theta, u)$.

Then, using (9) and (17), the estimation error dynamics satisfies the LPV system:

$$
\dot{\tilde{x}}=\left(\mathcal{A}(h(t))-K_{o} C_{2}\right) \tilde{x}
$$

Property (P4) implies that the parameter $h(t)$ evolves in a bounded set $\mathcal{H}$ whose vertex $\mathcal{V}$ is given by:

$$
\mathcal{V}=\left\{\alpha=\left(\alpha_{1}, \cdots, \alpha_{2 n+3}\right) \mid \alpha_{j} \in\left\{a_{j}, b_{j}\right\}\right\}
$$

From $(\mathbf{P} 4), \mathcal{V}$ reduces to $\mathcal{V}=\left\{a_{3}, b_{3}\right\}=\left\{0, \gamma-\frac{a+\beta_{3}}{m}\right\}$.

For all $P_{o}$ symmetric positive definite, a candidate Lyapunov function is given by:

$$
V_{o}(\tilde{x})=\tilde{x}^{T} P_{o} \tilde{x} \leq \bar{\lambda}\|\tilde{x}\|^{2}
$$

with $\bar{\lambda}$ the highest eigenvalue of $P_{o}$.

Differentiating (22) using (20) leads to:

$$
\dot{V}_{o}(\tilde{x})=\tilde{x}^{T} Q(h(t)) \tilde{x}
$$

with the time-varying matrix $Q(h)$ affine in $h$ defined by:

$Q(h(t))=\left(\mathcal{A}(h(t))-K_{o} C_{2}\right)^{T} P_{o}+\operatorname{Po}\left(\mathcal{A}(h(t))-K_{o} C_{2}\right)$

Let $K_{o}, P_{o}$ and $Q_{o}$ satisfy (18). Since $\mathcal{A}\left(a_{3}\right)=A_{1}$ and $\mathcal{A}\left(b_{3}\right)=A_{2}$, we consequently have $Q(\alpha)<-Q_{o} \forall \alpha \in \mathcal{V}$. 
Using the principle of convexity, we then have $Q(h(t))<$ $-Q_{o}, \forall h \in \mathcal{H}$. Hence, we have

$$
\dot{V}_{o}(\tilde{x})<-\tilde{x}^{T} Q_{o} \tilde{x}
$$

Let $\underline{\lambda}$ denote the smallest eigenvalue of $Q_{o}$, we then have

$$
\dot{V}_{o}(\tilde{x})<-\underline{\lambda}\|\tilde{x}\|^{2} \leq-(\underline{\lambda} / \bar{\lambda}) V_{o}(x)
$$

It follows from (26) that (17) is an exponential observer for system (9).

\section{B. Controller}

Proposition 2 Under assumptions of Lemma (1), the backstepping control law $u=\kappa(x)$ :

$$
\kappa(x)=-\frac{\left(k_{2}+k_{1}\right) \bar{z}_{2}+\left(1-k_{1}^{2}\right) \bar{z}_{1}+\phi(x)-\ddot{z}_{r}}{\beta_{2}}
$$

stabilizes the system (7) along any $\mathcal{C}^{0}$ reference trajectory for any bounded initial state $x(0)$ with the controller gains $k_{1}, k_{2}>0$ and $\bar{z}_{1}=z_{1}-z_{r}, \bar{z}_{2}=z_{2}+k_{1} z_{1}-\dot{z}_{r}$.

Proof: Let $\bar{z}_{1}=z_{1}-z_{r}$ and $\bar{z}_{2}=z_{2}-\dot{z}_{r}-\alpha$ for some stabilizing function $\alpha$.

- A candidate Lyapunov function is:

$$
V_{1}=\frac{1}{2} \bar{z}_{1}^{2} \Longrightarrow \dot{V}_{1}=\bar{z}_{1}\left(z_{2}-\dot{z}_{r}\right)=\bar{z}_{1}\left(\bar{z}_{2}+\alpha\right)
$$

Setting $\alpha=-k_{1} \bar{z}_{1}$ leads to:

$$
\dot{V}_{1}=-k_{1} \bar{z}_{1}^{2}+\bar{z}_{1} \bar{z}_{2}
$$

Since $\dot{\bar{z}}_{1}=z_{2}-\dot{z}_{r}=\bar{z}_{2}+\alpha=\bar{z}_{2}-k_{1} \bar{z}_{1}$, we obtain:

$$
\dot{\bar{z}}_{2}=\phi(x)+\beta_{2} u+k_{1}\left(\bar{z}_{2}-k_{1} \bar{z}_{1}\right)-\ddot{z}_{r}
$$

- In this last step, the candidate Lyapunov function is:

$$
V_{2}=V_{1}+\frac{1}{2} \bar{z}_{2}^{2}
$$

Differentiating (31) using (30), we obtain:

$$
\dot{V}_{2}=-k_{1} \bar{z}_{1}^{2}+\bar{z}_{2}\left(\phi(x)+\beta_{2} u+k_{1} \bar{z}_{2}+\left(1-k_{1}^{2}\right) \bar{z}_{1}-\ddot{z}_{r}\right)
$$

Using $u=\kappa(x)$ given by (27) leads to:

$$
\dot{V}_{2}=-k_{1} \bar{z}_{1}^{2}-k_{2} \bar{z}_{2}^{2}
$$

\section{Stability of the observer-based controller}

Proposition 3 Under assumptions of Proposition (1), the observer-based control law $\hat{u}=\kappa(\hat{x})$ :

$$
\kappa(\hat{x})=-\frac{\left(k_{2}+k_{1}\right) \hat{\bar{z}}_{2}+\left(1-k_{1}^{2}\right) \hat{\bar{z}}_{1}+\phi(\hat{x})-\ddot{z}_{r}}{\beta_{2}}
$$

stabilizes the system (7) along any $\mathcal{C}^{0}$ reference trajectory for any initial state $(x(0), \hat{x}(0)) \in \mathcal{K}^{2}$ with the controller gains $k_{1}, k_{2}>0, \hat{\bar{z}}_{1}=\hat{z}_{1}-z_{r}, \hat{\bar{z}}_{2}=\hat{z}_{2}+k_{1} \hat{z}_{1}-\dot{z}_{r}$ and $\tilde{z}=z-\hat{z}$.
Proof: Replacing $u$ with $\hat{u}$ in (32), we obtain:

$$
\dot{V}_{2}=-k_{1} \bar{z}_{1}^{2}-k_{2} \bar{z}_{2}^{2}+\bar{z}_{2}\left(\tilde{\phi}+\left(k_{1}+k_{2}\right) \tilde{\bar{z}}_{2}+\left(1-k_{1}^{2}\right) \tilde{\bar{z}}_{1}\right)
$$

with $\tilde{\phi}=\phi(x)-\phi(\hat{x})$. Using property (P1) leads to:

$$
\begin{aligned}
\dot{V}_{2} & \leq-k_{1}\left\|\bar{z}_{1}\right\|^{2}-k_{2}\left\|\bar{z}_{2}\right\|^{2}+\gamma\left\|\bar{z}_{2}\right\|\|\tilde{x}\| \\
& +\left(k_{1}+k_{2}\right)\left\|\bar{z}_{2}\right\|\left\|\tilde{z}_{2}\right\|+\left(1-k_{1}^{2}\right)\left\|\bar{z}_{2}\right\|\left\|\tilde{z}_{1}\right\|
\end{aligned}
$$

Yet we have the following bound:

$$
\begin{aligned}
\bar{z}_{2}=z_{2}+k_{1} \bar{z}_{1} & \Longrightarrow \tilde{\bar{z}}_{2}=\tilde{z}_{2}+k_{1} \tilde{\bar{z}}_{1} \\
& \Longrightarrow\left\|\tilde{\bar{z}}_{2}\right\| \leq\left\|\tilde{z}_{2}\right\|+k_{1}\left\|\tilde{\bar{z}}_{1}\right\|
\end{aligned}
$$

So using (37) in (36) yields:

$$
\begin{aligned}
\dot{V}_{2} & \leq-k_{1}\left\|\bar{z}_{1}\right\|^{2}-k_{2}\left\|\bar{z}_{2}\right\|^{2}+\gamma\left\|\bar{z}_{2}\right\|\|\tilde{x}\| \\
& +\left(k_{1}+k_{2}\right)\left\|\bar{z}_{2}\right\|\left\|\tilde{z}_{2}\right\|+\left(1+k_{1} k_{2}\right)\left\|\bar{z}_{2}\right\|\left\|\tilde{z}_{1}\right\| \\
& \leq-\eta_{c}\|\bar{z}\|^{2}+\zeta_{c}\|\bar{z}\|\|\tilde{x}\|
\end{aligned}
$$

with $\eta_{c}=\min \left(k_{1}, k_{2}\right)$ and $\zeta_{c}=\left(1+\gamma+k_{1}+k_{2}+k_{1} k_{2}\right)$.

Consider the Lyapunov function $W(\bar{z}, \tilde{x})$ with $\zeta>0$ :

$$
W(\bar{z}, \tilde{x})=\zeta V_{2}(\bar{z})+V_{o}(\tilde{x})
$$

Using (38) and (26), the time derivative of $W(\bar{z}, \tilde{x})$ is:

$$
\dot{W}(\bar{z}, \tilde{x}) \leq-\zeta \eta_{c}\|\bar{z}\|^{2}+\zeta \zeta_{c}\|\bar{z}\|\|\tilde{x}\|-\underline{\lambda}\|\tilde{x}\|^{2}
$$

Choosing $\zeta=\eta_{c} \underline{\lambda} / \zeta_{c}^{2}$ thus results in:

$$
\dot{W}(\bar{z}, \tilde{x}) \leq-\zeta \eta_{c}\|\bar{z}\|^{2} / 2-\underline{\lambda}\|\tilde{x}\|^{2} / 2
$$

Set $\kappa_{1}=\max (\zeta / 2, \bar{\lambda})$ and $\kappa_{2}=\min \left(\zeta \eta_{c}, \underline{\lambda}\right) / 2$, we get:

$$
\begin{aligned}
& W(\bar{z}, \tilde{x}) \leq \kappa_{1}\left(\|\bar{z}\|^{2}+\|\tilde{x}\|^{2}\right) \\
& \dot{W}(\bar{z}, \tilde{x}) \leq-\kappa_{2}\left(\|\bar{z}\|^{2}+\|\tilde{x}\|^{2}\right)
\end{aligned}
$$

We consequently have:

$$
\dot{W}(\bar{z}, \tilde{x}) \leq-\frac{\kappa_{2}}{\kappa_{1}} W(\bar{z}, \tilde{x})
$$

Therefore $\bar{z}$ and $\tilde{x}$ exponentially converge to zero, and the same goes for $z$ and $\tilde{x}$.

TABLE I

PARAMETERS VALUES

\begin{tabular}{|c||c|c|}
\hline Blood viscosity & $\eta$ & $16 \times 10^{-3}[\mathrm{~Pa} . \mathrm{s}]$ \\
\hline Blood density & $\rho_{f}$ & $1060\left[\mathrm{~kg} \cdot \mathrm{m}^{-3}\right]$ \\
\hline Ferromagnetic density & $\rho_{m}$ & $7500\left[\mathrm{~kg} \cdot \mathrm{m}^{-3}\right]$ \\
\hline Robot radius & $r$ & $2.510^{-4}[\mathrm{~m}]$ \\
\hline Vessel diameter & $D$ & $310^{-3}[\mathrm{~m}]$ \\
\hline Payload density & $\rho_{p}$ & $1500\left[\mathrm{~kg} \cdot \mathrm{m}^{-3}\right]$ \\
\hline Ferromagnetic ratio & $\tau_{m}$ & 0.75 \\
\hline Magnetization & $M$ & $1.23 \times 10^{6}\left[\mathrm{A.m} \mathrm{m}^{-1}\right]$ \\
\hline Controller gains & $\left(k_{1}, k_{2}\right)$ & $(7,14)$ \\
\hline
\end{tabular}



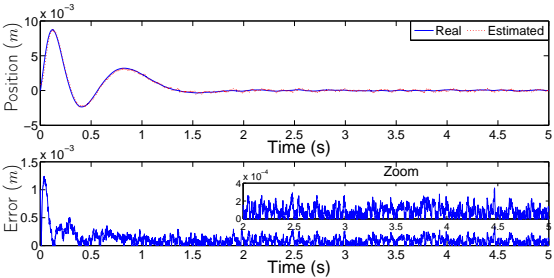

(a) Robot position and position estimation error

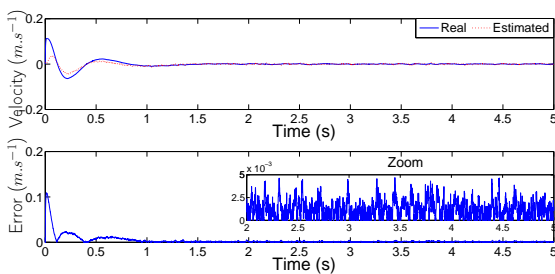

(d) Robot velocity and velocity estimation error

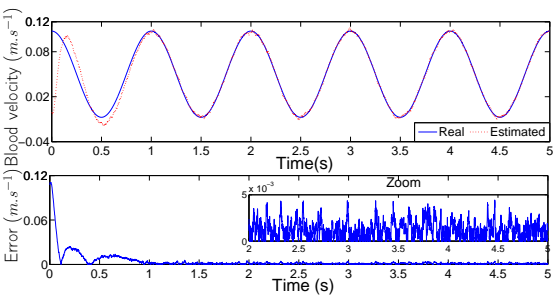

(g) Blood velocity and its estimation error

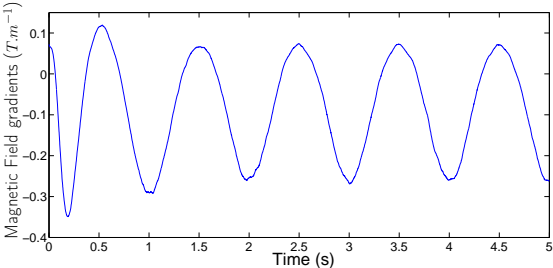

(j) Control input: $\nabla B$

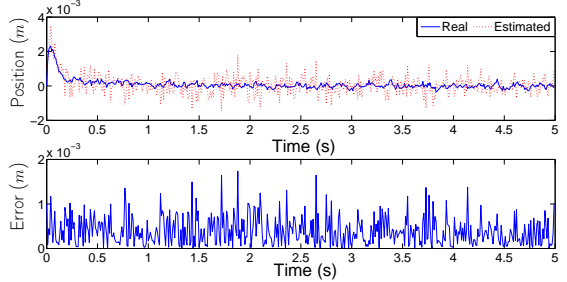

(b) Robot position and position estimation error

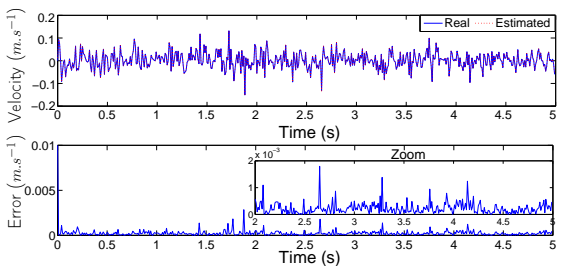

(e) Robot velocity and velocity estimation error

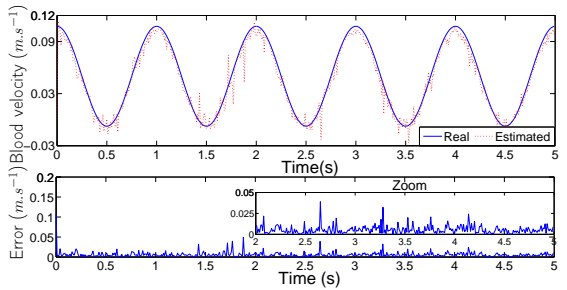

(h) Blood velocity and its estimation error

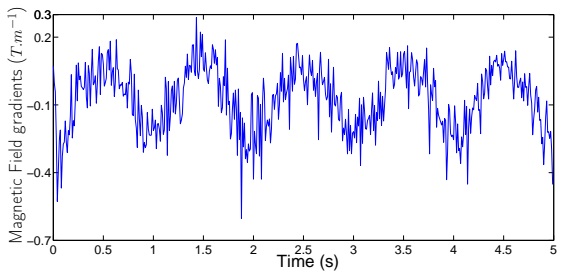

(k) Control input: $\nabla B$.

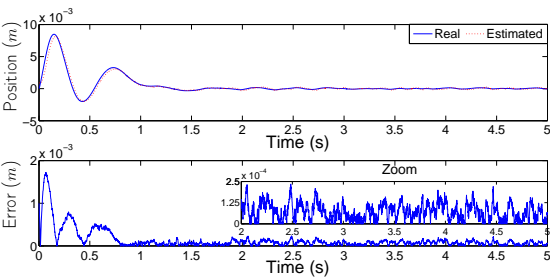

(c) Robot position and position estimation error

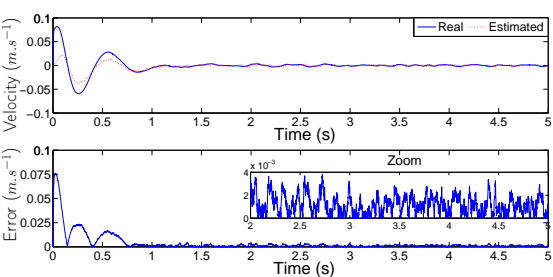

(f) Robot velocity and velocity estimation error

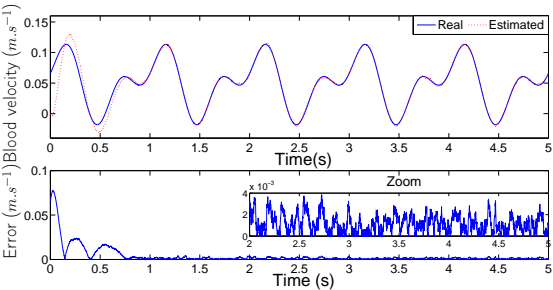

(i) Blood velocity and its estimation error

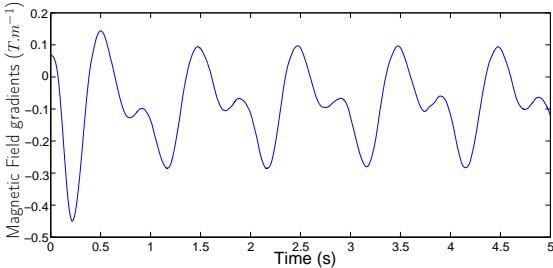

(1) Control input: $\nabla B$

Fig. 3. From left to right: simulation with MVT-observer $n=1$, simulation with high gain observer $n=1$, and simulation with MVT-observer $n=2$. From top to bottom: robot position, robot velocity, blood velocity, and control input.

TABLE II

INITIAL CONDITIONS AND MVT OBSERVER GAINS

\begin{tabular}{|c|c|c|}
\hline \multirow{3}{*}{$n=1$} & $x_{0}$ & $(000.107500 .05)$ \\
\cline { 2 - 3 } & $\hat{x}_{0}$ & $(0.0010 .0100 .0010 .01)$ \\
\cline { 2 - 3 } & $K_{o}$ & $(68.091340 .761051 .073561 .35549 .92)$ \\
\hline \multirow{3}{*}{$n=2$} & $x_{0}$ & $(000.06590 .35660 .1239-0.80820 .2)$ \\
\cline { 2 - 3 } & $\hat{x}_{0}$ & $(0.0010 .0100 .0010 .0010 .0010 .01)$ \\
\cline { 2 - 3 } & $K_{o}$ & $(33.0579 .1566 .83337 .0276 .4-4039.41296 .4)$ \\
\hline
\end{tabular}

\section{Simulations}

In the simulations, a Gaussian white noise with a standard deviation of $500 \mu \mathrm{m}$ is added to the measured output to model the medical imagers resolution. The nominal values of parameters are given in Table I. The robot is bead pulled, so that we have $\beta_{2}=\frac{\tau_{m} M}{\rho}$ and $\beta_{3}=0$. Without loss of generality, the reference trajectory is a fixed point.

The first set of simulations compares the results of the proposed approach with results of the high gain observer developped in [15] (with a gain $L=3$ ) for a blood velocity modeled by a first-order Fourier series. For the second set of simulations, the blood velocity is modeled by a secondorder Fourier series. The high gain is so high that the innovation terms dwarf the observer dynamics and amplify the noise tremendously: we address this simulation only for the observer detailed in Proposition (1). Table II gives the initial conditions and the MVT observer gains.

\section{A. First-order truncated blood velocity $(n=1)$}

The first simulation shows that the MVT-observer-based controller is stable despite the output noise. The Fig. 3(a) (resp. 3(d)) illustrates the real and estimated microrobot positions (resp. velocities) and the associated estimation errors. The microrobot position and velocity converge to zero after a $1 s$ long transient phase that corresponds to the observer convergence time. Once stabilized the position and velocity estimation errors are less than $400 \mu \mathrm{m}$ and $5 \mathrm{~mm} . \mathrm{s}^{-1}$, respectively. Similarly, the Fig. 3(g) shows that the blood velocity estimation error is less than $5 \mathrm{~mm} . \mathrm{s}^{-1}$ 
once the observer has converged. The Fig. 3(j) illustrates that the control input is not affected by noise.

The second simulation illustrates the results obtained with a high gain observer and the same controller than in the previous simulation. The Fig. 3(b) (resp. 3(e)) illustrates the real and estimated microrobot positions (resp. velocities) and the associated estimation errors. The high gain converges twice more quickly than the MVT observer due to high values of the innovation terms. Yet the price to pay is a higher sensitivity to noise, which is noticeable on Fig. 3(b) and 3(h): the position estimation error is around $2 \mathrm{~mm}$ and the blood velocity estimation error is around $50 \mathrm{~mm} . \mathrm{s}^{-1}$. The high gain observer performs better than the MVT observer only for the microrobot velocity estimation, with an error less than $2 m m . s^{-1}$. The control input is far more affected by noise (Fig. 3(k)) than in the previous simulation.

\section{B. Second-order truncated blood velocity $(n=2)$}

The third simulation illustrates the results obtained for $n=$ 2 using the MVT observer-based controller. The convergence rate of the observer is the same than for $n=1$. The estimation errors on the microrobot position, velocity and on the blood velocity are respectively less than $250 \mu \mathrm{m}$, $4 m m . s^{-1}$ and $4 m m . s^{-1}$ (see Fig. 3(c)-3(i)). The observer here gives better results than for $n=1$ because the gains on the first three components are smaller than in the case $n=1$. One can notice that the shape of the second order velocity shown on Fig. 3(i) is very close to the real profile of blood velocity (see Fig. 2).

\section{DISCUSSION AND CONCLUSION}

Measurement of average blood flow velocity has been largely addressed in the literature. Yet such information is neither sufficient nor accurate enough for controlling microrobots in blood vessels. The pulsatile blood velocity affects nonlinearly the drag force, which in turn affects the microrobot dynamics. We take advantage of the Fourier series decomposition of the blood velocity to propose an observer-based controller using the sole measurement of the microrobot position obtained thanks to an imager. Simulations illustrate the improved robustness to noise of the MVT based observer with respect to the high gain observer we have previously developed.

Extension of the approach to a $2 \mathrm{D}$ model is an ongoing work. The main difficulty is induced by the increased Lipschitz constant of a system including the electrostatic force. The estimation of the blood pulsation is still an open issue, since the resulting perturbation model becomes nonlinear and non uniformly observable. Kazantsis-Kravaris Luenberger observer has been tested, but the resulting estimated state is not yet suitable for control purpose.

This approach can not only benefit to the control of magnetic microrobots, but also as an alternative to direct measurement of the blood velocity. It may also merge the two advantages, e.g. for a removal of the plaques in clogged arteries followed by an inspection of the blood velocity changes so as to check if the plaques have been removed.

\section{REFERENCES}

[1] B. J. Nelson, I. K. Kaliakatsos, and J. J. Abbott, "Microrobots for minimally invasive medecine," Annual Review of Biomedical Engineering, vol. 12, pp. 55-85, 2010.

[2] G. Kosa, M. Shoham, and M. Zaaroor, "Propulsion method for swimming microrobots," IEEE Transactions on Robotics, vol. 23(1), pp. 137-150, 2007.

[3] M. C. Lagomarsino, F. Capuani, and C. P. Lowe, "A simulation study of the dynamics of a driven filament in an aristotelian fluid," Journal of Theoretical Biology, vol. 224(2), pp. 215-224, 2003.

[4] A. A. Evans and E. Lauga, "Propulsion by passive filaments and active flagella near boundaries," Physical Review E, vol. 82(4), p. 041915, 2010.

[5] R. Dreyfus, J. Beaudry, M. L. Roper, M. Fermigier, H. A. Stone, and J. Bibette, "Microscopic artificial swimmers," Nature, vol. 437, pp. 862-865, 2005.

[6] J. Edd, S. Payen, B. Rubinsky, M. L. Stoller, and M. Sitti, "Biomimetic propulsion for a swimming surgical microrobot," IEEE International Conference on Intelligent Robots and Systems, Las Vegas, pp. 25832588,2003

[7] L. Zhang, K. E. Peyer, and B. J. Nelson, "Artificial bacterial flagella for micromanipulation," Lab on a chip, vol. 10, no. 17, pp. 2203-2215, 2010.

[8] J. J. Abbott, K. E. Peyer, M. C. Lagomarsino, L. Zhang, L. X. Dong, I. K. Kaliakatsos, and B. J. Nelson, "How should microrobots swim?" International Journal of Robotics Research, vol. 28, pp. 1434-1447, 2009.

[9] J.-B. Mathieu, G. Beaudoin, and S. Martel, "Method of propulsion of a ferromagnetic core in the cardiovascular system through magnetic gradients generated by an mri system," IEEE Transactions on Biomedical Engineering, vol. 53, no. 2, pp. 292-299, 2006.

[10] L. Arcese, M. Fruchard, and A. Ferreira, "Endovascular magneticallyguided robots: navigation modeling and optimization," IEEE Transactions on Biomedical Engineering, vol. 59(4), pp. 977-987, 2012.

[11] P. Vartholomeos and C. Mavroidis, "In silico studies of magnetic microparticle aggregations in fluid environments for mri-guided drug delivery," IEEE Transactions on Biomedical Engineering, vol. 59(11), pp. 3028-3038, 2012.

[12] K. W. Ferrara, B. G. Zagar, J. B. Sokil-Melgar, R. H. Silverman, and I. M. Aslanidis, "Estimation of blood velocity with high frequency ultrasound," IEEE Transactions on Ultrasonics, Ferroelectrics and Frequency Control, vol. 43(1), pp. 149-157, 1996.

[13] R. Ponzini, C. Vergara, G. Rizzo, A. Veneziani, A. Roghi, A. Vanzulli, O. Parodi, and A. Redaelli, "Womersley number-based estimates of blood flow rate in doppler analysis: In vivo validation by means of phase-contrast mri," IEEE Transactions on Biomedical Engineering, vol. 57(7), pp. 1807-1815, 2010.

[14] C. S. Kim, C. Kiris, D. Kwak, and T. David, "Numerical simulation of local blood flow in the carotid and cerebral arteries under altered gravity," Journal of Biomechanical Engineering, vol. 128(2), pp. 194202, 2006.

[15] M. Fruchard, L. Arcese, and E. Courtial, "Estimation of the blood velocity for nanorobotics," IEEE Transactions on Robotics, vol. 30, no. 1, pp. 93-102, 2014.

[16] A. Krener and W. Kang, "Locally convergent nonlinear observers," SIAM, vol. 42, no. 1, pp. 155-177, 2003.

[17] F. White, Viscous Fluid Flow. McGraw Hill New-York, 1991.

[18] R. Kehlenbeck and R. D. Felice, "Empirical relationships for the terminal settling velocity of spheres in cylindrical columns," Chemical Eng. Technology, vol. 21, pp. 303-308, 1999.

[19] J. Womersley, "Method for the calculation of velocity, rate of flow and viscous drag in arteries when the pressure gradient is known," The Journal of physiology, vol. 127(3), pp. 553-563, 1955.

[20] L. Arcese, M. Fruchard, and A. Ferreira, "Adaptive controller and observer for a magnetic microrobot," IEEE Transactions on Robotics, vol. 29(4), pp. 1060-1067, 2013.

[21] L. M. Silverman and H. E. Meadows, "Controllability and observability in time-variable linear systems," SIAM Journal on Control and Optimization, vol. 5, pp. 64-73, 1967.

[22] A. Isidori, Nonlinear control systems. Springer-Verlag London, 1995.

[23] A. Zemouche and M. Boutayeb, "On lmi conditions to design observers for lipschitz nonlinear systems," Automatica, vol. 49, no. 2, pp. $585-591,2013$. 